\section{Vietnam Journal of Agricultural Sciences}

\title{
Generation of Synthetic Peptide-Specific Antibody for the Development of A Southern Rice Black-Streaked Dwarf Virus Diagnostic Test
}

\author{
Do Thi Hanh ${ }^{1}$, Nguyen Anh Minh², Nguyen Van $\mathrm{Cuu}^{2}$, Phung Thi \\ Thu Huong ${ }^{2}$, Pham Xuan $\mathrm{Hoi}^{2}$ \& Nguyen Duy Phuong ${ }^{2}$
}

${ }^{1}$ Faculty of Chemical Technology, Hanoi University of Industry, Hanoi 129000, Vietnam 2Department of Molecular Pathology, Agricultural Genetics Institute, Hanoi 123100, Vietnam

\begin{abstract}
Southern rice black-streaked dwarf virus (SRBSDV) causes severe epidemical disease on rice with the infected area up to millions of hectares in South China and North and Central of Vietnam. So far, there are no effective, cheap, quick, and practicable methods for diagnosing SRBSDV. The conventional RT-PCR technique is the most popular method for detecting SRBSDV with high accuracy. However, it is hard to apply this method for large-scale SDBSDV diagnosis because of the requirements of expensive reagents and instruments, as well as complex procedures. Meanwhile, SRBSDV diagnostic techniques based on antigen detection have outstanding advantages due to their low cost, easy manipulation, and wide application possibility. Today, there are still no commercially available specific antibodies to SRBSDV. In a previous study, to develop the SRBSDV diagnostic technique by the ELISA technique, a SRBSDV specific antibody was generated by a recombinant P10 envelope protein $(66 \mathrm{kDa})$, which has a titer of 1:5,000. In this study, we continued to study the production of SRBSDV specific polyclonal antibodies from small antigen-rich peptides from the SRBSDV P10 envelope protein. The resulting purified antibody can specifically bind to the P10 protein and at the diluted concentration of 1:100,000 it can detect SRBSDV in infected rice samples via the dot-blot technique. Our research results open up new opportunities for proactive antibodies to develop a SRBSDV membrane rapid diagnostic kit.
\end{abstract}

\section{Keywords}

Polyclonal antibody, rice, P10 envelope protein, SRBSDV

Received: May 18, 2020 Accepted: July 19, 2021

\section{Correspondence to} phuongnd.bio@gmail.com

\section{Introduction}

Southern rice black-streaked dwarf virus (SRBSDV), a member of the genus Fijivirus in the family Reoviridae, is one of the most 
dangerous harmful rice viruses and is distributed mainly in southern Asia including China, Japan, and Vietnam (Guo et al., 2008; Zhang et al., 2008; Ngo Vinh Vien et al., 2009; Cuong et al., 2010; Wang et al., 2010; Matshukura et al., 2013). SRBSDV was first observed in Vietnam in 2009 and caused severe epidemical disease in huge rice-growing areas in North and Central Vietnam. So far, the development of an SRBSDV-resistant rice variety is not yet available; therefore, disease management is still primarily aimed at controlling the source of the pathogen, especially the disease vector-like white-backed planthopper (Sogatella furcifera).

The genome of SRBSDV is about 29,124bp in length, consisting of 10 double-stranded RNA segments (from S1 to S10), ranging in size from 1.8 to $4.5 \mathrm{~kb}$. In particular, the segment $\mathrm{S} 10$ has a length of $1,797 \mathrm{bp}$, containing 1,674 nucleotides and an ORF encoding proteins that form the outer shell of the virus molecule with a corresponding molecular weight of $62.6 \mathrm{kDa}$. The $\mathrm{P} 10$ protein is highly conservative, and has been identified as having specific interactions with the vectors of SRBSDV; therefore, it plays an important role in the evolution of the virus. For this reason, the P10 protein is often chosen as the target for developing SRBSDV diagnostic methods (Yin et al., 2011).

Currently, the most common SRBSDV diagnostic technique is reverse transcriptionpolymerase chain reaction (RT-PCR) using specific primers designed based on a highly conservative sequence on the S10 segment of the viral genome (Zhang et al., 2008; Ngo Vinh Vien et al., 2009; Cuong et al., 2010; Anh et al., 2011). SRBSDV diagnostic techniques using real-time RT-PCR are also being applied to quantify viruses for early diagnosis (Zhang et al., 2013). Chinese laboratories have produced antiSRBSDV antibodies using several synthetic peptide fragments of the P10 protein and developed a diagnostic technique using dotELISA (Wang et al., 2012).

In previous studies, we have successfully created polyclonal antibodies against SRBSDV from the recombinant P10 envelope protein segment to detect SRBSDV in ELISA tests (Do Thi Hanh et al., 2015). However, in order to develop an SRBSDV rapid diagnostic kit, the antibodies produced need to be specific and have a high titer to ensure the accuracy and sensitivity of the diagnostic kit. In this research, we continued to generate the polyclonal antibodies against SRBSDV based on small conservative antigen-rich peptides derived from the P10 protein of representative Vietnam SRBSDV isolates. The resulting antibody will be the premise for developing an SRBSDV rapid diagnostic kit in future.

\section{Materials and Methods}

\section{Materials}

The SRBSDV artificially infected rice samples, with and without visible symptoms that showed positive results in RT-PCR tests, were supported by the Research Center for Tropical Plant Pathology, Vietnam National University of Agriculture. Laboratory 8-week-old mice were ordered from the National Institute of Hygiene and Epidemiology. The recombinant peptide was synthesized by the GeneCreate Company (China).

\section{Methods}

\section{Preparation of bacterium and rice extracts}

Small-scale extractions were conducted to test for dot blotting and western blotting experiments. For the rice extraction, approximately 1.0 gram of fresh rice leaf tissue was ground into a powder in liquid nitrogen and homogenized with $1.0 \mathrm{~mL}$ of $1 \mathrm{X}$ PBS buffer. For the bacterium extraction, the recombinant $E$. coli cell lines containing either the pET28a or pET28a/P10 vector were cultured for protein expression as previously described (Pham Thanh Tam et al., 2013), and then lysated in 1X PBS buffer by using a sonicator device. All mixtures were then centrifuged at $16,000 \times \mathrm{g}$ for $10 \mathrm{~min}$ and the supernatant was kept as a testing sample.

\section{Design of the antigenic peptide fragment}

The conservative amino acid sequence on the P10 protein of SRBSDV was identified by BioEdit 2.0 software based on the published S10 segment sequences of the Vietnam SRBSDV isolates (Nguyen Hoang Quang et al., 2013). The 
hydrophilic property and surface probability of the amino acids on the P10 protein molecule were analyzed by the Antibody Epitope Prediction tool (http://tools.immuneepitope.org/). The antigenicity of the P10 protein molecule was analyzed by the Antibody Epitope Prediction tool (http://tools.immuneepitope.org/) and the Predicting Antigenic Peptides tool. The antigen epitope was predicted by the Antibody Epitope Prediction tool (http://tools.immuneepitope.org/) and the BepiPred-2.0 tool (http://www.cbs.dtu.dk/).

\section{Immunization of mice with the synthetic peptide}

Immunization experiments were conducted as previously described (Amero et al., 1994). Mice were immunized with $20.0 \mu \mathrm{g}$ of purified fused peptide mixed with $200.0 \mu \mathrm{L}$ of PBS buffer (50mM potassium phosphate, $150 \mathrm{mM} \mathrm{NaCl}, \mathrm{pH}$ 7.25) and an equal volume of Freund's Complete/Incomplete Adjuvant. The mixture was injected into mice at the peritoneal abdomen. Three immunizations were delivered intraperitoneally, administered one week apart. Tail bleeds were performed to check for immunization responses before primary immunization and one week after each reimmunization with a dot blotting assay (Sambrook \& Russell, 2001). When a mouse achieved the highest immune response, the entire mouse blood was collected to extract the serum.

\section{Purification of the IgG antibody}

The IgG antibodies in the mouse serum were purified by a protein A-sepharose affinity chromatography column (Thermo Fisher Scientific) as described by Amero et al. (1994). The mouse antisera were titrated to $\mathrm{pH} 8.0$ by adding 0.1 volumes of $1 \mathrm{M}$ Tris- $\mathrm{HCl}(\mathrm{pH} 8.0)$. The column was equilibrated with $10.0 \mathrm{~mL}$ of 100 $\mathrm{mM}$ Tris- $\mathrm{HCl}$ buffer ( $\mathrm{pH} 8.0$ ). After loading the sample, the column was washed, respectively, with $100 \mathrm{mM}$ and $10 \mathrm{mM}$ Tris- $\mathrm{HCl}$ buffer. The target protein was eluted by $100 \mathrm{mM}$ Glycin- $\mathrm{HCl}$ buffer ( $\mathrm{pH}$ 3.0). The eluted solution was neutralized to $\mathrm{pH} 8.0$ by adding 0.1 volumes of 1 $\mathrm{M}$ Tris- $\mathrm{HCl}$ buffer ( $\mathrm{pH} 8.0$ ). The purity of the IgG antibody was confirmed by SDS-PAGE gel electrophoresis and western blotting analysis (Sambrook \& Russell, 2001).

\section{Western blotting and dot blotting assays}

Recombinant proteins in purified and unpurified samples were detected via western blotting assays (Sambrook \& Russell, 2001). The protein samples, after being separated by the electrophoresis method on an SDS-PAGE gel, were transferred electronically to a PVDF membrane in Tris-glycine buffer with $20 \%$ methanol. The membrane was immobilized by gently shaking it (for 30 to 60 minutes) in $1 \%$ BSA solution and incubated alternately with primary and secondary antibodies for 1 hour. The targeted protein was colored by incubation in $\mathrm{p}$ nitro blue tetrazolium chloride 5-bromo-4chloro-3-indolyl phosphate (NBT/BCIP) substrate solution.

For the dot blotting assay, the PVDF membrane was activated by soaking it in methanol for 10 seconds. Solutions containing the target protein (prepared in 1X PBS buffer) were dropped directly onto the membrane. Membrane blocking, antibody probing, and detection steps were similar to the western blotting assay (Sambrook \& Russell, 2001).

\section{Results and Discussion}

\section{Design of the peptide antigen}

The P10 protein of SRBSDV is a common target in diagnostic assays of SRBSDV using specific antibodies (Zhang et al., 2013; Do Thi Hanh et al., 2015). To create antibodies specifically binding to the P10 protein of Vietnamese SRBSDV isolates, the peptide fragment antigen was screened according to whether it was located on the (i) antigenic or (ii) conservative region of the $\mathrm{P} 10$ protein. In a previous study, we evaluated the S10 genetic diversity of SRBSDV isolates collected from rice growing in different ecological areas of Vietnam (Nguyen Hoang Quang et al., 2013). Based on those published sequences, the conservative region on the P10 protein of SRBSDV isolated in Vietnam was identified (Figure 1).

The antigenic regions (epitopes) of a protein consist of residues located on the surface of the protein structure that are capable of causing an immune response and thus, determine the quality 


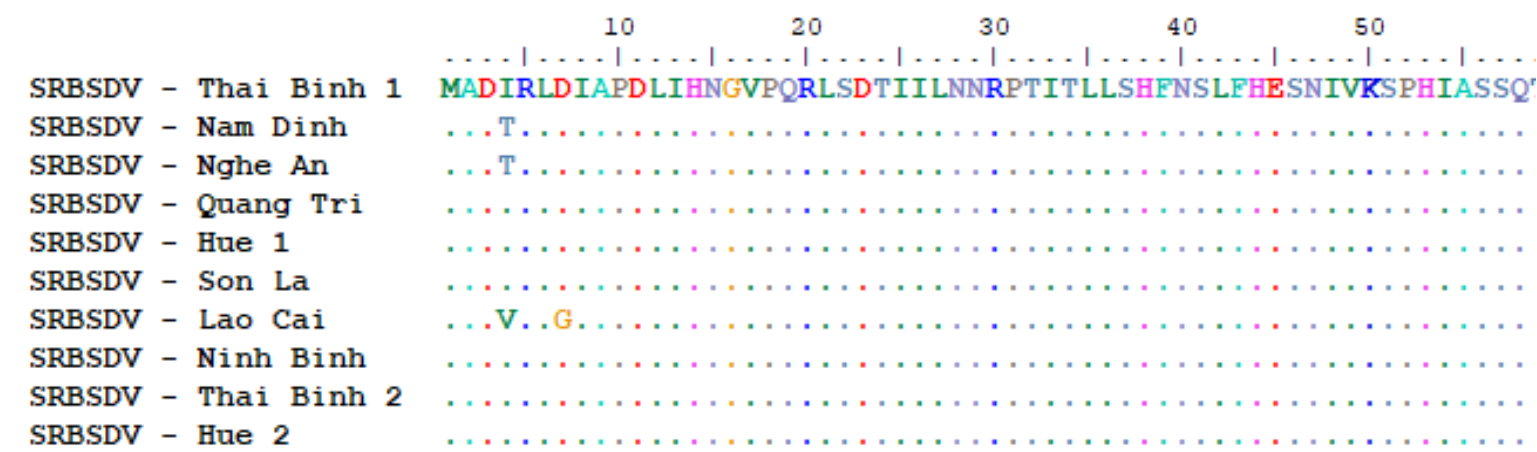

Note: Parts of the deductive amino acid sequences from the P10 protein of ten Vietnamese SRBSDV isolates (Thai Binh 1, Nam Dinh 1, Nghe An, Quang Tri, Hue 1, Son La, Lao Cai, Ninh Binh, Thai Binh 2, and Hue 2) were analyzed by BioEdit 2.0 software. The dots (.) indicate the same amino acid.

Figure 2. The deductive amino acid sequence of the $\mathrm{P} 10$ protein

of the generated antibody. Currently, the bioinformatics tools supporting epitope prediction are very diverse, increasingly being developed in both quantity and quality, and are used in the diagnosis of pathogenic viruses not only in plants but also in humans. Antigenic peptides on the P10 envelope protein were predicted by using the Antibody Epitope Prediction, Predicting Antigenic Peptides, and BepiPred-2.0 tools (Figure 2). The analysis showed that the peptide fragment (NGVPQRLSDTIILNNRP) located on the Nterminus of the P10 protein, at position 15-31, (named P10[15-31]) met all the requirements of an epitope, including (i) having the right concentration of hydrophilic amino acids(Figure 2A), (ii) being capable of having a location on the molecular surface (Figure 2B), (iii) being capable of creating epitopes (Figure 2C), (iv) having high antigenicity (Figure 2D), and (v) being located on the conservative region of the protein P10. This peptide sequence was selected and artificially synthesized for the mice immunization experiment.

\section{Immunization of mice}

A small peptide is often complex for the immune system of animals to recognize when it enters the body. Therefore, to enhance antigenspecific immunity, the peptide segment P10[1531] was fused with a keyhole limpet hemocyanin $(\mathrm{KLH})$-carrying protein in combination with adjuvant supplementation in the immunization of the mice. After four immunization injections, mouse blood was collected for the extraction of the serum. The presence of antibodies against the P10 protein in the antisera was tested by using the dot blot technique.

The results showed that the antiserum of non-immunized mice had no reaction with purified P10 protein (Figure 3A, column 4), as well as the extraction of SRBSDV infected rice plants (Figure 3A, columns 1 and 3). After immunization, antiserum (with a 1000 dilution) showed positive results in dot blotting tests of both the P10 protein (Figure 3B, column 4) and SRBSDV infected rice (Figure 3B, column 2) samples. There was no cross-reactivity between the antisera of the immunized mice and the SRBSDV non-infected rice extract (Figure 3B, column 3). This analysis allowed us to initially conclude that the generation of polyclonal antibodies against the P10 protein using the peptide antigen $\mathrm{P} 10[15-31]$ in laboratory mice was successful; and the archived antiserum contained antibodies against the P10 protein of SRBSDV.

\section{Purification of anti-P10 IgG antibodies}

Although the archived antisera contained antibodies that recognized the P10 protein, there were actually many other undesirable proteins in the serum. Therefore, the A-sepharose protein affinity column chromatography system was used to eliminate these unwanted proteins in order to obtain purified IgG antibodies. The optical density (OD) measurement result of the eluted fractions showed that most of the adsorbed proteins ( $\mathrm{IgG}$ antibody) were concentrated in three fractions, namely E6, E7, and E8, with a very high peak on the chromatogram (Figure 4A). 
Generation of synthetic peptide-specific antibody for the development of a SRBSDV diagnostic test

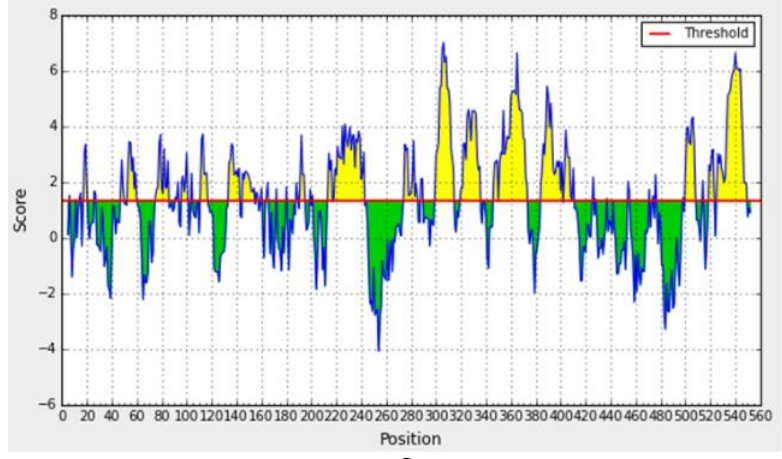

A

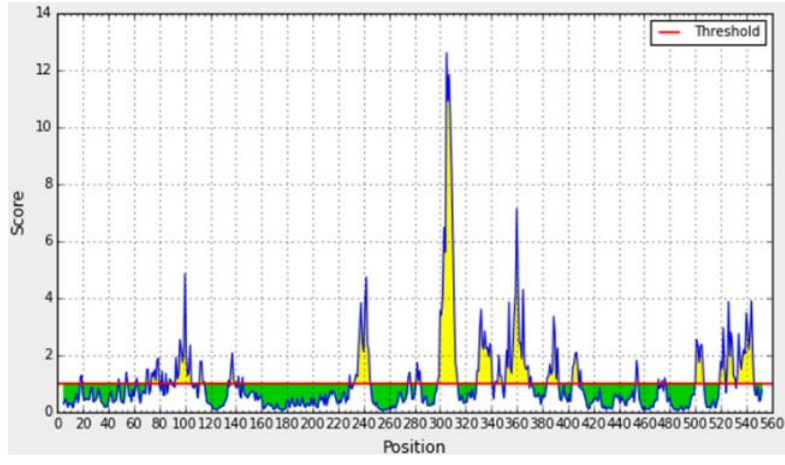

B

\begin{abstract}
Epitopes : $\ldots \ldots \ldots$. EEEEEEEEEEEEEEEE. EEE.............EEEEE. EEEEEEEEEEE.....
...EEEEEEEEEEEEEEEEEEEEEEEEEEEEEEEEEEEEEEEEE.

... EEEEEEEEEEEEEEEEEEEEEEEEEE Predictions: MADIRLDIAPDLIHNGVPQRLSDTIILNNRPTITLLSHFNSLFHESNIVKSPHIASSPTTVNLYIRKHLLTRLHDRLQTVETSTLPNITQLKEHIQSYFRNEHQPIFQTLTNNNLSDEFLGVTTFGLSLFATSKLDAEQIERVQIETLTEGNVTLKPFSADG

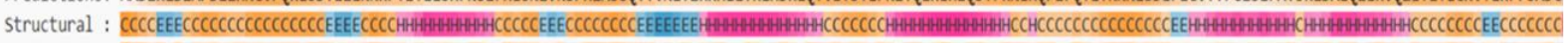

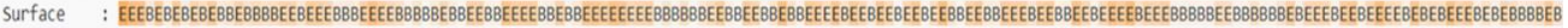

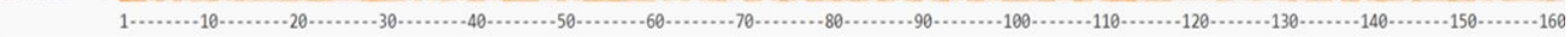

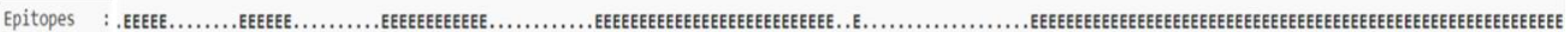
Predictions: .EVILDDSYIGIIGKISGLEVHKLLDKCCREVPAQMGILTDEVKLLIRSGKLRIDGGYDFNCPASTTDVTHYGGYDQYSRQMFEKLNLFFNISLSIIPVSALKTIHVFEKELSALDADKSLLEQTWSGVSSFIETWKVKTKAKDEDQDEYELTGLSALRKGVD Structural : \#HHHCCCHHHHHHCCCCCHHHHHHHHHHCCCCCCCCCCHCEEEEECCEEEECCCCCCCCCCCCCCCCCCCCCCHHHHHHHHHHHHHCEEEEEEEHHHHHHCCCHHHHHHCCCCCHHHHHCCCCCCEEEEEEHHHHCCCCCCCHHHHHHHHHHCCCCC

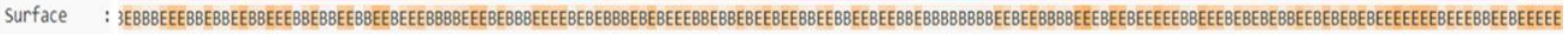

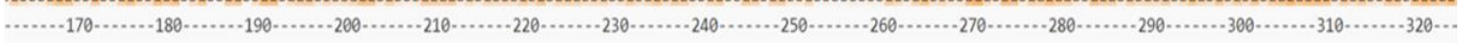

\title{
C
}

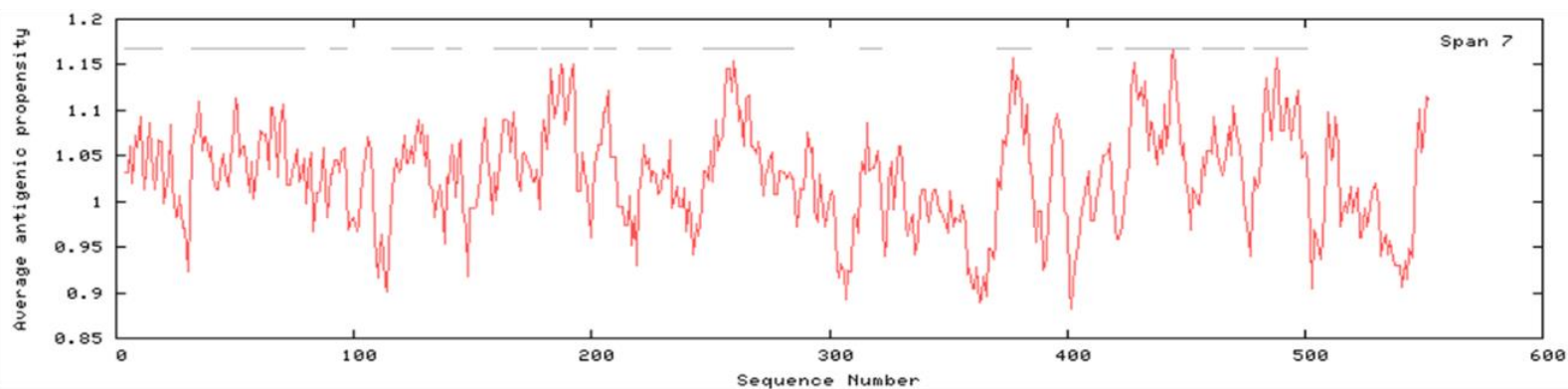

D

Note: The Hydrophilicity $(A)$ and Surface Accessibility $(B)$ were analyzed by the Antibody Epitope Prediction tool; the red line represents the threshold value. The epitopes and molecular structure of the P10 protein (C) were predicted by the BepiPred 2.0 tool; the letter $E$ represents the position of the epitope. The antigenicity of the P10 protein $(D)$ was analyzed by the Predicting Antigenic Peptides tool; the bar represents the amino acid zone with high antigenicity.

Figure 3. Prediction of the antigenicity of the P10 protein

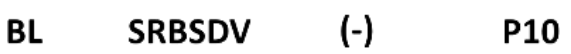

A

B

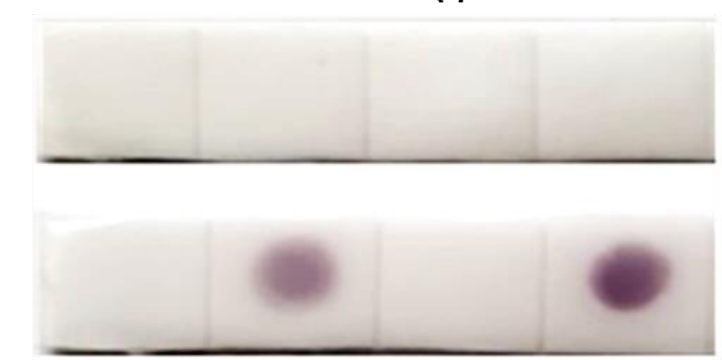

Note: Pre-immunization $(A)$ and 4-week immunization (B) antisera were diluted at 1:1000. (BL) Blank control (PBS buffer);

(SRBSDV): SRBSDV-infected rice extract; (-): Healthy rice extract; (P10) Purified P10 protein.

Figure 4. Evaluation of the $\mathrm{P} 10$-specific antiserum responses in mice 


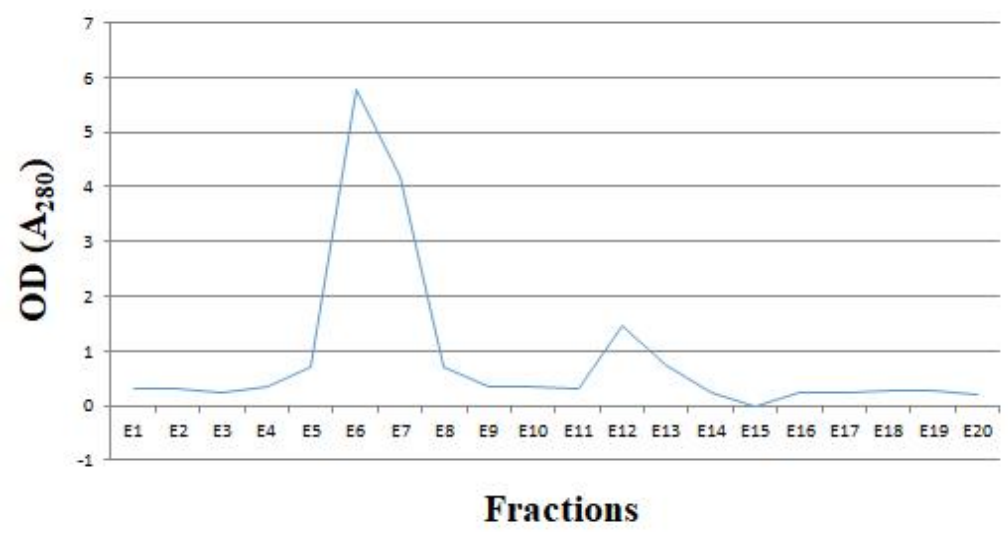

A

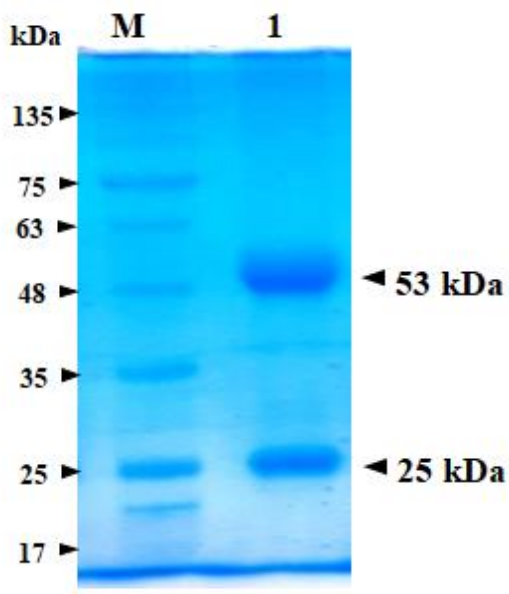

B

Note: (A) $O D_{280 n m}$ analysis of the chromatographic fractions; (B) SDS-PAGE analysis of the purified lgG antibody; lane M: protein ladder (iNtRon); lane 1: E7 fraction of the purified IgG antibody.

Figure 5. Purification of IgG antibodies using protein A-sepharose affinity column chromatography

To confirm the purity of the obtained antibody, the specific binding protein fraction obtained from the A-sepharose protein column (fraction 7) was analyzed by using SDS-PAGE electrophoresis. There were two protein bands shown on the SDS-PAGE gel with the sizes of about 25 and $53 \mathrm{kDa}$ (Figure 4B, lane 1), corresponding to the sizes of the light chain and the heavy chain, respectively, of the $\operatorname{IgG}$ antibody. This result proved that the $\operatorname{IgG}$ antibody had been purified successfully from mouse serum using the A-sepharose protein affinity chromatography system.

\section{Titer and specificity evaluation of the antibody}

In order to determine the titer of the archived antibody, the dot blotting test was conducted with both the SRBSDV-infected rice extraction and the purified recombinant $\mathrm{P} 10$ protein sample, using a range dilution of the $\mathrm{IgG}$ antibody (from $1: 10,000$ to $1: 100,000)$. The results of the dot blotting test showed that the purified $\operatorname{IgG}$ antibody could detect the target protein at the $1: 100,000$ dilution and 1:80,000 dilution in the purified protein sample and SRBSDV-infected rice extract sample, respectively (Figure 5, column "P10" and "SRBSDV").

To assess the specificity of the polyclonal IgG antibody, a western blotting assay was conducted on the $1: 10,000$ dilution of the purified antibody, using a total lysate of the P10 expression in E. coli Rossetta cells (Pham Thanh Tam et al., 2013) as a testing sample. The results of the western blotting analysis showed that our antibody could identify a single approximately $66 \mathrm{kDa}$ band in the E. coli extract sample (Figure 6B, lane 1), as well as in the purified $\mathrm{P} 10$ protein sample (Figure 6B, lane 3). In contrast, there was no protein band in the negative control experiment using the crude extract of $E$. coli Rossetta cells carrying empty pET28a vectors (Figure 6B, lane 2). These results proved that our purified IgG antibodies could specifically recognize the recombinant P10 protein of SRBSDV.

To confirm the potential of antibody application in SRBSDV diagnosis, purified IgG antibody (diluted at 1:10,000) was subjected to a dot blotting test against the different rice extracts containing SRBSDV. Colored dots were clearly observed for all the SDBSDV-infected rice samples that showed obvious symptoms (Figure 7, S1 - S4). In contrast, the healthy sample did not exhibit a colored spot (Figure 7, uninfected). In the case of the SRBSDV-infected rice samples without apparent symptoms (Figure 7, NS1 NS3), they showed lighter spots. These results indicated that the polyclonal anti-P10[15-31] peptide antibody could be used to diagnose SRBSDV. 
Generation of synthetic peptide-specific antibody for the development of a SRBSDV diagnostic test

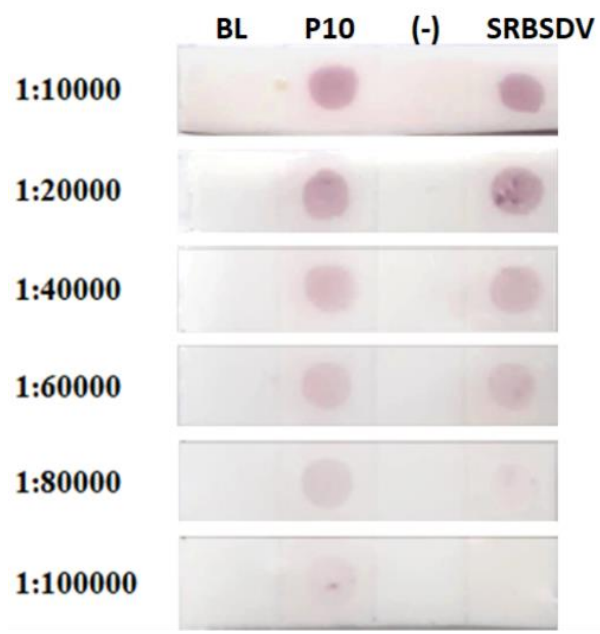

Note: Purified IgG antibody was diluted at 1:10,000, 1:20,000, 1:40,000, 1:60,000, 1:80,000, and 1:100,000. (BI) Blank control (PBS buffer); (P10) Purified P10 protein; (-) Negative control (Healthy rice extract); (SRBSDV) SRBSDV-infected rice extract.

Figure 6. Titer of the purified polyclonal lgG antibody against the $\mathrm{P} 10$ protein

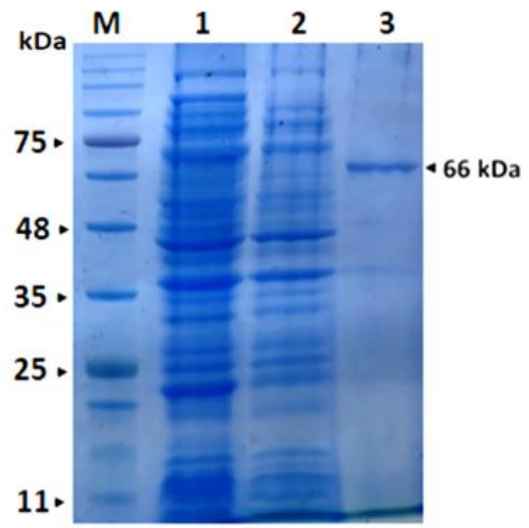

A

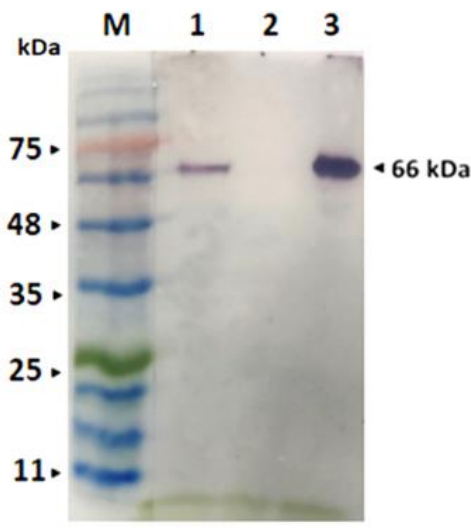

B

Note: (A) SDS-PAGE analysis of the protein samples. (B) Western blotting detection of the recombinant $P 10$ protein using purified IgG antibody at a 1:10,000 dilution. Lane M: protein ladder (iNtRon); lane 1: extract of pET28a/P10 E. coli Rossetta cells; lane 2: extract of pET28a/P10 E. coli Rossetta cells (negative control); lane 3: purified P10 protein.

Figure 7. Specificity evaluation of the purified IgG antibody

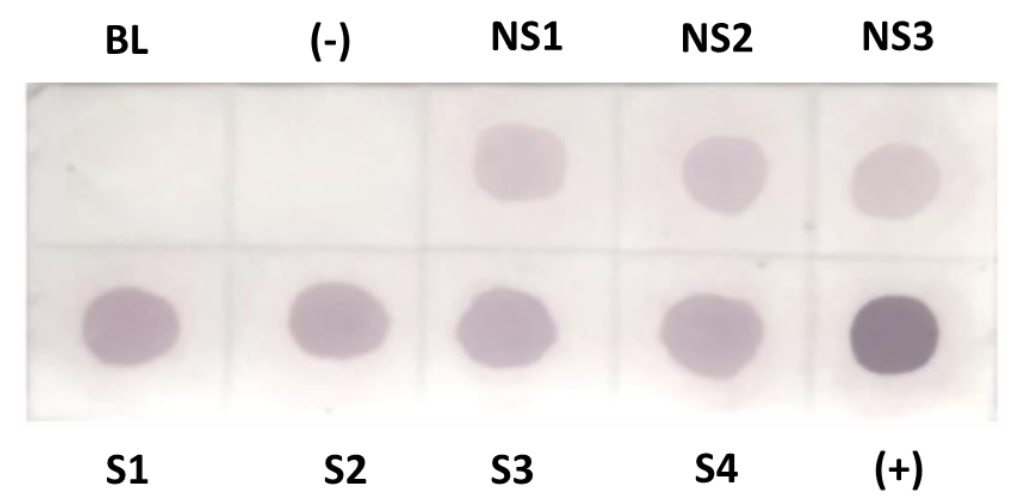

Note: Purified IgG antibody was diluted at 1:10,000. (BL) blank control (PBS buffer); (-) negative control (healthy rice extract); (NS1 - NS3) non-symptomatic SRBSDV-infected rice extract; (S1 - S4) symptomatic SRBSDV-infected rice extract; (+) positive control (purified P10 protein).

Figure 8. Diagnosis of SRBSDV infected rice samples using purified IgG antibody 
So far, only Wang et al. (2012) have been successful in the production of specific antiSRBSDV antibodies by using three peptides with lengths of 20 amino acids, respectively, located at 20-39, 140-159, and 319-339 on the sequence of the P10 protein. The obtained antibody had a titer of 1:500,000 in the ELISA assays. In a previous study, we also used the full-length P10 protein of SRBSDV for mouse immunization and archived IgG antibodies with a titer of 1:6,000. In this study, the anti-SRBSDV antibody was created by a synthetic peptide fragment located at the 15-31 position on the P10 protein, which showed a titer of 1:100,000 in the dot blotting assays. Our antibody detected the presence of SRBSDV in both non-symptomatic and symptomatic rice samples. The archived antibody had a lower titer than that mentioned in the publication of Wang et al., but this could be due to the different titer evaluation methods. Wang et al. (2012) used an ELISA assay for tittering the antibody, in which the measured results were based on the $\mathrm{OD}_{405 \mathrm{~nm}}$ value. It is likely that the presence of chlorophyll in the rice samples affected the ELISA results. Therefore, they used the antibody at a dilution of 1:1,500 for the dot blotting diagnosis, and this antibody has not yet been commercialized. Our research results are a significant step in the development of a rapid diagnostic test kit for SRBSDV detection in Vietnam.

\section{Conclusions}

A P10[15-31] peptide fragment with a length of 16 amino acids was identified as having high antigenicity on the envelope of the $\mathrm{P} 10$ protein of SRBSDV. Polyclonal IgG antibodies against P10[15-31] were successfully generated from laboratory mice and purified via protein Asepharose column chromatography. The purified antibody had a titer of 1:100,000, and could specifically recognize the recombinant P10 protein in bacterial cell extracts and in SRBSDV in both symptomatic and non-symptomatic SRBSDV-infected rice samples by using dot-blot at the dilution of 1:10,000.

\section{Acknowledgments}

This research was under the project entitled "Development of the production technology for Southern rice black-streaked dwarf virus diagnosis KIT" (2019-2020) which was funded by the Ministry of Agriculture and Rural Development.

\section{References}

Amero S. A., James T. C. \& Elgin C. R. (1994). Production of antibodies using protein in gel bands. Basic Protein and Peptide Protocols (Humana Press, editor). Springer. 32: 717-720.

Anh T. H., Zhang H. M., Yang J., Chen J. P., Hebrard E., Zhou G. H., Vien N. V. \& Cheng J. A. (2011) Identification, characterization, and distribution of southern rice black-streaked dwarf virus in Vietnam. Plant Disease. 95: 1063-1069.

Cuong H. V., Hai N. V., Man V. T. \& Masaru M. (2010). Rice dwarf disease in North Vietnam in 2009 is caused by southern rice black-streaked dwarf virus (SRBSDV). Bulletin of the Institute of Tropical Agriculture, Kyushu University: 85-92.

Guo H. Z., Jung W. J., Jiang C. D., Peng L., Lin X. D. \& Guang Z. S. (2008). Southern rice black-streaked dwarf virus: A new proposed Fijivirus species in the family Reoviridae. Chinese Science Bulletin. 53: 3677-3685.

Do Thi Hanh, Pham Thi Van \& Pham Xuan Hoi (2015). Generation of polyclonal antibody against SRBSDV by recombinant protein. Vietnam Journal Agriculture and Rural Development. 278(1): 38-43 (in Vietnamese).

Matshukura K., Towata T., Sakai J., Onuki M. \& Matsumura M. (2013). Dynamics of southern rice black-streaked dwarf virus in rice and implication for virus acquisition. Journal of Phytopathology. 103: 509-512.

Nguyen Hoang Quang, Do Thi Hanh, Pham Thi Van, Tran Thi Nhu Hoa, Ha Viet Cuong \& Pham Xuan Hoi (2013). Sequence analysis of S10 segment of virus isolates causing black-streaked dwarf disease on rice in Vietnam. Journal of Vietnam Agricultural Science and Technology. 2: 26-31 (in Vietnamese).

Sambrook J. \& Russell D. W. (2001). Molecular Cloning: A Laboratory Manual ( $3^{\text {rd }}$ ed.). Cold Spring Harbor Laboratory. Cold Spring Harbor, NY.

Pham Thanh Tam, Pham Thi Van, Nguyen Hoang Quang, Nguyen Duy Phuong \& Pham Xuan Hoi (2013). Expression and purification of outer capsid protein P10 of Southern Rice Black-Striked Dwarf virus. Vietnam Journal Agriculture and Rural Development. 231(2): 35-40 (in Vietnamese).

Ngo Vinh Vien, Pham Thi Vuong, Nguyen Nhu Cuong, Ta Hoang Anh, Nguyen Thi Me, Phan Bich Thu, Pham 
Hong Hien \& Ha Viet Cuong (2009). Diagnostic results for rice black-streak dwarf virus in some northern provinces of Vietnam. Journal of Plant Protection. 6: 8-18 (in Vietnamese).

Wang Q., Yang J., Zhou G. H., Zhang H. M., Chen J. P. \& Adams M. J. (2010). The complete genome sequence of two isolates of southern rice black-streaked dwarf virus, a new member of the genus Fijivirus. Journal of Phytopathology. 158: 733-737.

Wang Z., Yu D., Li X., Zeng M., Chen Z., Bi L., Liu J., Jin L., Hu D., Yang S. \& Song B. (2012). The development and amplification of a Dot-ELISA assay for diagnosis of southern rice black-streaked dwarf virus in the field. Viruses. 4: 167-183.
Yin X., Xu F., Zheng F., Li X., Liu B. \& Zhang C. (2011). Molecular characterization of segments S7 to S10 of a southern rice black-streaked dwarf virus isolate from maize in Northern china. Virologica Sinica. 26(1): 47-53.

Zhang H. M., Yang J., Chen J. P. \& Adams M. J. (2008). A black-streaked dwarf disease on rice in China is caused by a novel fijivirus. Archives of Virology. 153: 18931898.

Zhang S., Zhang D., Liu Y., Luo X., Cheng J. \& Peng J. (2013). Development of a real-time RT-PCR method for detection and qualification of southern rice blackstreaked dwarf virus in rice. Journal of Plant Pathology and Microbiology. 4(7): 1-4. 